\title{
Dynamic Changes of Liver Function Indexes in Patients with Different Clinical Types of COVID-19
}

\author{
Wei Zhao*, Xiaoqing Zhang*, Feng Zhu, Xiufeng Jiang \\ Department of Respiratory and Critical Care Medicine, Wuxi Fifth People's Hospital, Wuxi, 214000, People's Republic of China \\ *These authors contributed equally to this work \\ Correspondence: Feng Zhu; Xiufeng Jiang, Department of Respiratory and Critical Care Medicine, Wuxi Fifth People's Hospital, Wuxi, 2 I4000, \\ People's Republic of China, Tel +86-I39I4I35026; +86-I3013630369, Email 18I5I5688@qq.com; jiangxiufeng69@I63.com
}

\begin{abstract}
Background: To investigate the liver function indexes and dynamic changes in patients with different clinical types of new coronavirus pneumonia (COVID-19).

Methods: A retrospective analysis of 170 COVID-19 patients hospitalized in the Wuxi Fifth People's Hospital was divided into asymptomatic group (13 cases), mild-common group (142 cases) and seriously-critically ill group (15 cases), the clinical data and liver function indexes of the three groups were compared.

Results: A total of 170 patients included 94 males and 76 females, with an average age of $44.7 \pm 17.8$ years. Seriously-critically ill group was older, and the proportion of patients with diabetes and liver injury at admission was also higher. As the hospitalization time increased, the changes of alanine aminotransferase (ALT) levels in asymptomatic group and mild-common group were not significant (all P > 0.05), while the ALT levels of seriously-critically ill group showed a curve that first flattened and then decreased (degree of freedom: $1.809, \mathrm{P}=0.002$ ). Compared with the mild-common group, the daily decrease of ALT was $1.220 \mathrm{U} / \mathrm{L}$ more in the seriouslycritically ill group $(\mathrm{P}<0.001)$. The aspartate aminotransferase (AST) in asymptomatic group and seriously-critically ill group did not decrease significantly (all P > 0.05), while the AST in mild-common group decreased significantly (regression coefficient: -10.507 , $\mathrm{P}=0.008)$. There was no significant difference in AST changes between the three groups $(\mathrm{P}=0.250-0.904)$.
\end{abstract}

Conclusion: Liver injury is common in COVID-19 patients, especially for severe patients; the dynamic change pattern of liver function indicators may be helpful to judge liver injury and evaluate treatment effects in patients with different clinical types.

Keywords: COVID-19, liver injury, ALT, AST

\section{Introduction}

Since the new coronavirus pneumonia (COVID-19) was reported in December 2019, it has expanded rapidly worldwide, posing severe dangers to human health and great challenges to public health management. ${ }^{1}$ The main target organ of the new coronavirus (SARS-CoV-2) is the lung, and the main clinical manifestations are fever, fatigue, and dry cough. Most (81\%) patients are mild, with a good prognosis; some patients (14\%) are seriously ill; and a small number of patients $(5 \%)$ are critically ill, which can lead to the failure of multiple organs. ${ }^{2-4}$

In addition to respiratory symptoms, studies ${ }^{5-7}$ have shown that SARS-CoV-2 infection can cause other multi-organ diseases, including liver injury. There are many potential causes of liver injury in COVID-19 patients, such as cell injury caused by direct virus. SARS-CoV-2 can directly enter hepatocytes through angiotensin-converting enzyme 2 receptor (ACE2), expressed in liver and bile duct cells. ${ }^{8}$ Cell apoptosis, hepatocyte swelling, acidophilic bodies, and lobular inflammation found in liver biopsy specimens are considered the result of direct virus injury. ${ }^{9}$ In addition, the immunemediated excessive inflammatory response related to COVID-19 can also lead to liver injury, and inflammatory cytokine storms have been found in most severely ill patients. ${ }^{10}$ Some studies have pointed out that the levels of pro-inflammatory cytokines and chemokines in patients with liver dysfunction are higher than those in patients with normal liver function, ${ }^{11,12}$ which indicates a correlation between liver injury and inflammatory response in COVID-19 patients. 
Hypoxia and shock caused by COVID-19 related complications such as systemic inflammatory response syndrome, respiratory distress syndrome, and multiple organ injury may also lead to liver ischemia and hypoxia-reperfusion dysfunction. ${ }^{13-16}$ Furthermore, basic liver diseases such as chronic hepatitis B and liver cirrhosis can also lead to abnormally elevated liver enzymes, ${ }^{17}$ and the drugs used in the treatment of COVID-19 patients may also be related to liver injury. For example, the use of multiple medications, such as antibiotics, antiviral drugs, antipyretics, analgesics, and traditional Chinese medicines, may cause liver injury in patients. ${ }^{18,19}$

Studies have found that liver injury is mainly manifested as abnormal liver function indexes: about $14 \%-53 \%$ of COVID-19 patients showed abnormal levels of alanine aminotransferase (ALT) and aspartate aminotransferase (AST), ${ }^{20,21}$ and these indexes were more significantly elevated in severe patients. ${ }^{22}$ However, few reports describe the dynamic changes in liver injury indexes during patient hospitalization. In clinical practice, the time interval between liver function tests varies for each patient, and the baseline level of liver function alone cannot meet the needs of clinical diagnosis and treatment. Therefore, our study aims to examine the dynamic changes in liver function indexes in patients with different clinical types of COVID-19 and guide clinicians in early detection and monitoring of liver injury.

\section{Materials and Methods}

\section{General Information}

This study is a retrospective cohort study. We analyzed the data from COVID-19 patients who received treatment in the negative pressure ward of Wuxi Fifth People's Hospital from January 2020 to April 2020. This study followed the principles stipulated in the Declaration of Helsinki and was approved by the institutional ethics committee for retrospective analysis (No. 2020-015-1). Each patient signed written informed consent, and the patients under 18 signed informed consent by their legal guardian. Inclusion criteria: (1) Positive for COVID-19 nucleic acid detection via realtime fluorescent RT-PCR; (2) follows the diagnostic criteria of "COVID-19 Pneumonia Diagnosis and Treatment Plan (Trial Version 7)". ${ }^{23}$ Exclusion criteria: (1) pregnant women; (2) no complete medical history, especially no liver function tests during hospitalization. The general information, complications, biochemical indexes at admission, and liver function indexes across the hospitalization period of each patient were collected.

\section{Diagnosis and Treatment}

The clinical classification of COVID-19 patients is mainly based on symptoms and imaging features. According to the "COVID-19 Pneumonia Diagnosis and Treatment Plan (Trial version 7)" issued by the General Office of National Health Commission of China, ${ }^{23}$ the patients were divided into four types: mild, common, seriously ill, and critically ill. The clinical symptoms of mild patients are minor, and there is no pneumonia manifestation from imaging. The common type of patient has fever and signs in the respiratory tract and pneumonia manifestations from imaging. The seriously ill patient has one or more of the following symptoms: shortness of breath, $R R \geq 30$ times/min, oxygen saturation $\leq 93 \%$ at rest, arterial blood oxygen partial pressure/oxygen concentration $\leq 300 \mathrm{mmHg}$. The critically ill patient has one or more of the following conditions: respiratory failure and the requirement for mechanical ventilation; shock; combined with other organ failures that require ICU monitoring. The COVID-19 patients can be treated with antiviral therapies, such as $\alpha$-interferon, lopinavir/ritonavir, and ribavirin, together with effective and timely oxygen therapy. Chinese medicine can also be used on some occasions. In addition to symptomatic treatment for seriously and critically ill patients, it is also important to prevent complications, treat the underlying diseases, prevent secondary infections, and provide timely support for organ function. Silymarin will be used for patients with abnormal liver function.

\section{Laboratory Testing}

The laboratory test parameters were as follows: Alanine aminotransferase (ALT, reference range: 6-32U/L), aspartate aminotransferase (AST, reference range: 9-45U/L), total bilirubin (TBIL, reference range: 5-21umol/L), albumin (ALB, reference range: 40-55g/L), globulin (GLO, reference range: 20-40 g/L), glucose (GLU, reference range: 4.1-5.9 mmol/ L), blood urea nitrogen (BUN, Reference range: $1.25-5.29 \mathrm{mmol} / \mathrm{L}$ ), serum creatinine ( $\mathrm{SCr}$, reference range: $41-77 \mu \mathrm{mol} /$ L), blood potassium (K, reference range: $3.5-5.3 \mathrm{mmol} / \mathrm{L}$ ), blood sodium ( $\mathrm{Na}$, reference range: $137-147 \mathrm{mmol} / \mathrm{L}$ ), blood 
chlorine $(\mathrm{Cl}$, reference range: $96-108 \mathrm{mmol} / \mathrm{L})$. All the parameters were measured using BECKMAN COULTER AU5800 (Brea, CA, USA).

\section{Statistical Analysis}

Continuous variables were expressed as mean $\pm \mathrm{SD}$ or median (Q1-Q3), and categorical variables were expressed as frequency (\%). Unpaired Student's $t$-test or Mann Whitney nonparametric test was used to compare continuous variables. Pearson chi-square test and Fisher's exact test were used to analyze categorical variables.

Using linear regression, we analyzed the changes in patient ALT and AST levels with hospitalization time. Next, the Generalized Additive Mixed Model (GAMM) was used to examine the changes in ALT and AST with hospitalization time in patients with different disease classifications. GAMM can eliminate the effect of individual variation on repeated measurements by introducing random factors in statistical analysis. ${ }^{24}$ The linear and non-linear relationships between ALT/AST changes and hospitalization time were examined and were adjusted for potential confounding factors, such as sex, age, hypertension, diabetes, coronary heart disease, cerebrovascular disease, tumor, hepatitis B virus (HBV), chronic kidney disease (CKD), chronic liver disease, smoking history, alcoholism, etc. R 3.4 .3 (http://www.r-project.org) was used for all analyses. $\mathrm{P}<0.05$ was considered statistically significant, and all statistical tests were two-tailed.

\section{Results}

A total of 170 COVID-19 patients (including 13 asymptomatic patients) were involved in this study, including 94 males and 76 females, with an average age of $44.7 \pm 17.8$ years (range: 5-91 years). Besides asymptomatic patients, the remaining 157 patients were divided into 37 mild cases, 105 common cases, 13 seriously ill cases, 2 critically ill cases (1 patient changed from mild to seriously ill at 2 days after admission, so this patient was classified as seriously ill). Based on the clinical significance, the patients were further divided into asymptomatic group (13 cases), mild-common group (142 cases), and seriously-critically ill group (15 cases).

The general information of the three groups and the comparison of liver function and biochemical indexes at admission are shown in Table 1. The patients in seriously-critically ill group were older than that of the other two groups $(\mathrm{P}<0.05)$, and the proportion of patients with diabetes mellitus was significantly higher than that of the asymptomatic group (33.3\% vs $0 \%, \mathrm{P}<0.05)$, but there was no significant difference in the proportion of hypertension with the other two groups. The ALT in the seriously-critically ill group was higher than that in the other two groups, but the difference was not significant $(\mathrm{P}>0.05)$, AST was significantly higher than that in the mild-common group at admission $(\mathrm{P}<0.05)$, and the proportion of liver injury in the seriously-critically ill group was significantly higher than that in the mild-common group $(53.3 \%$ vs $8.5 \%, \mathrm{P}<0.05)$. In addition, compared with the mild-common group, the blood glucose was higher, and albumin was lower in seriously-critically ill group (all $\mathrm{P}<0.05$ ); compared with the asymptomatic group, the blood sodium and chlorine in the seriously-critically ill group was lower (all $\mathrm{P}<0.05$ ).

\section{The Changes in ALT and AST with Hospitalization in All Patients}

After adjusting for clinical classification, sex, age, hypertension, and diabetes, we found that the ALT levels of all patients stabilized first and then gradually decreased as hospitalization time prolonged (degree of freedom: 1.893, $\mathrm{P}=0.014$ ) (Figure 1A). Moreover, the AST levels of all patients linearly reduced as time increased (regression coefficient: $-13.228,95 \%$ CI: $-19.856--6.601, \mathrm{P}<0.001$ ) (Figure 1B).

\section{The Changes in ALT and AST in Patients with Different Clinical Types}

The asymptomatic group has a shorter hospital stay (approximately 15 days) than the other two groups. After adjusting for sex, age, hypertension, and diabetes, we found that, as the hospitalization time increased, the ALT levels of asymptomatic group decreased, but the change was not significant (regression coefficient: $-55.034,95 \%$ CI: -110.564-0.496, $\mathrm{P}=0.053$ ), the ALT levels of mild-common group slightly increased but not yet significant (regression coefficient: $6.821,95 \% \mathrm{CI}:-3.249-16.891, \mathrm{P}=0.185$ ), while the ALT levels of seriously-critically ill group showed a curve that was first flat and then decreased (degree of freedom: 1.809, $\mathrm{P}=0.002$ ) (Figure 2A). When compared with the asymptomatic group, there was no significant difference in the daily ALT changes between the mild-common group and 
Table I The General Information, Liver Function, and Biochemical Indexes of the Three Groups at Admission

\begin{tabular}{|c|c|c|c|c|}
\hline Combined Classification & $\begin{array}{l}\text { Asymptomatic } \\
\text { Group }\end{array}$ & $\begin{array}{l}\text { Mild-Common } \\
\text { Group }\end{array}$ & $\begin{array}{l}\text { Seriously-Critically III } \\
\text { Group }\end{array}$ & P-value \\
\hline $\mathbf{N}$ & 13 & 142 & 15 & \\
\hline Sex & & & & 0.540 \\
\hline Female & 7 (53.8\%) & $64(45.1 \%)$ & $5(33.3 \%)$ & \\
\hline Male & $6(46.2 \%)$ & 78 (54.9\%) & $10(66.7 \%)$ & \\
\hline Age & $40.1 \pm 15.1$ & $43.5 \pm 17.7$ & $60.2 \pm 14.6 * \#$ & 0.001 \\
\hline Grouping & & & & 0.070 \\
\hline$<60$ & $13(100.0 \%)$ & 117 (82.4\%) & $10(66.7 \%)$ & \\
\hline$\geq 60$ & $0(0.0 \%)$ & $25(17.6 \%)$ & $5(33.3 \%)$ & \\
\hline Hypertension & $7(53.8 \%)$ & $31(21.8 \%)^{*}$ & 5 (33.3\%) & 0.030 \\
\hline Diabetes & $0(0.0 \%)$ & $20(14.1 \%)$ & $5(33.3 \%)^{*}$ & 0.040 \\
\hline Coronary heart disease & $0(0.0 \%)$ & $5(3.5 \%)$ & $0(0.0 \%)$ & 1.000 \\
\hline Cerebrovascular disease & $0(0.0 \%)$ & I (0.7\%) & I (6.7\%) & 0.303 \\
\hline Tumor & $0(0.0 \%)$ & $3(2.1 \%)$ & $0(0.0 \%)$ & 1.000 \\
\hline HBV & $0(0.0 \%)$ & I (0.7\%) & $0(0.0 \%)$ & 1.000 \\
\hline CKD & $0(0.0 \%)$ & I (0.7\%) & I (6.7\%) & 0.305 \\
\hline Chronic liver disease/cirrhosis & I (7.7\%) & $2(1.4 \%)$ & $0(0.0 \%)$ & 0.233 \\
\hline Alcoholism & $0(0.0 \%)$ & $5(5.2 \%)$ & $0(0.0 \%)$ & 1.000 \\
\hline Smoking & $0(0.0 \%)$ & 7 (7.3\%) & $0(0.0 \%)$ & 0.655 \\
\hline \multicolumn{5}{|c|}{$\begin{array}{l}\text { Liver function and biochemical indicators at } \\
\text { admission }\end{array}$} \\
\hline ALT (U/L) & $30.0(26.5-69.5)$ & $20.0(14.5-26.1)$ & $48.0(15.0-71.0)$ & 0.056 \\
\hline AST $(\mathrm{U} / \mathrm{L})$ & $24.0(21.0-53.5)$ & $22.0(18.5-28.5)$ & $39.0(28.0-49.0) \#$ & 0.007 \\
\hline Total bilirubin (umol/L) & $9.0(7.5-13.0)$ & $7.7(5.0-13.9)$ & $9.0(7.0-12.0)$ & 0.866 \\
\hline Albumin $(g / L)$ & $42.6 \pm 3.7$ & $43.1 \pm 5.4$ & $36.6 \pm 4.3 \#$ & 0.003 \\
\hline Globulin (g/L) & $28.4 \pm 3.0$ & $28.0 \pm 6.4$ & $29.3 \pm 2.8$ & 0.826 \\
\hline Glucose (mmol/L) & $5.6(5.4-6.2)$ & $5.4(4.8-6.1)$ & $8.0(6.0-11.6) \#$ & 0.006 \\
\hline BUN (mmol/L) & $4.2(3.3-5.5)$ & $4.1(3.6-5.2)$ & $4.6(3.3-5.9)$ & 0.982 \\
\hline Creatinine (umol/L) & $56.5(49.0-76.0)$ & $65.0(48.5-78.5)$ & $64.0(55.0-73.0)$ & 0.863 \\
\hline Potassium (mmol/l) & $4.0 \pm 0.5$ & $4.0 \pm 0.5$ & $3.6 \pm 0.4$ & 0.073 \\
\hline Sodium (mmol/l) & $141.9 \pm 1.6$ & $139.0 \pm 3.7$ & $137.6 \pm 4.3^{*}$ & 0.045 \\
\hline Chlorine (mmol/l) & $105.6 \pm 1.1$ & $103.0 \pm 3.3$ & $101.4 \pm 5.0^{*}$ & 0.038 \\
\hline Liver injury & $4(30.8 \%)$ & $12(8.5 \%)^{*}$ & 8 (53.3\%)\# & $<0.001$ \\
\hline
\end{tabular}

Notes: The above results are expressed as Mean \pm SD/Median (QI-Q3)/N (\%); *there was a significant difference compared with asymptomatic group; ${ }^{*}$ there was a significant difference compared with mild-common group.

Abbreviations: HBV, hepatitis B virus; CKD, chronic kidney disease; ALT, alanine aminotransferase; AST, aspartate aminotransferase; BUN, blood urea nitrogen.

the seriously-critically ill group ( $\mathrm{P}=0.253$ and 0.928$)$; however, compared with the mild-common group, we found that the daily decrease in ALT was 1.220 (95\% CI: $0.562-1.879)$ more in the seriously-critically ill group, and the difference was statistically significant $(\mathrm{P}<0.001)$ (Figure $2 \mathrm{~A})$.

For the AST levels, after adjusting for sex, age, hypertension, and diabetes, we found that asymptomatic group and the seriously-critically ill group showed decreased AST as hospitalization time increased, but the change was not significant (regression coefficient: -35.091 and $-13.366,95 \%$ CI: $-78.474-8.292$ and $-29.283-2.552, \mathrm{P}=0.114$ and 0.100 ), while the mild-common group showed a significantly decreased (regression coefficient: $-10.507,95 \%$ CI: $-18.293--2.722, \mathrm{P}=0.008$ ) (Figure $2 \mathrm{~B}$ ). In addition, there was no significant difference in daily AST changes between the three groups $(\mathrm{P}=0.250-0.904)$ (Figure $2 \mathrm{~B})$.

\section{Discussion}

$\mathrm{Su}$ and $\mathrm{Kao}^{2}$ reported that older people and patients with underlying diseases (such as diabetes, hypertension) had a higher risk of severe COVID-19. Kukla et $\mathrm{al}^{25}$ suggested that diabetic patients were more susceptible to SARS-CoV-2 

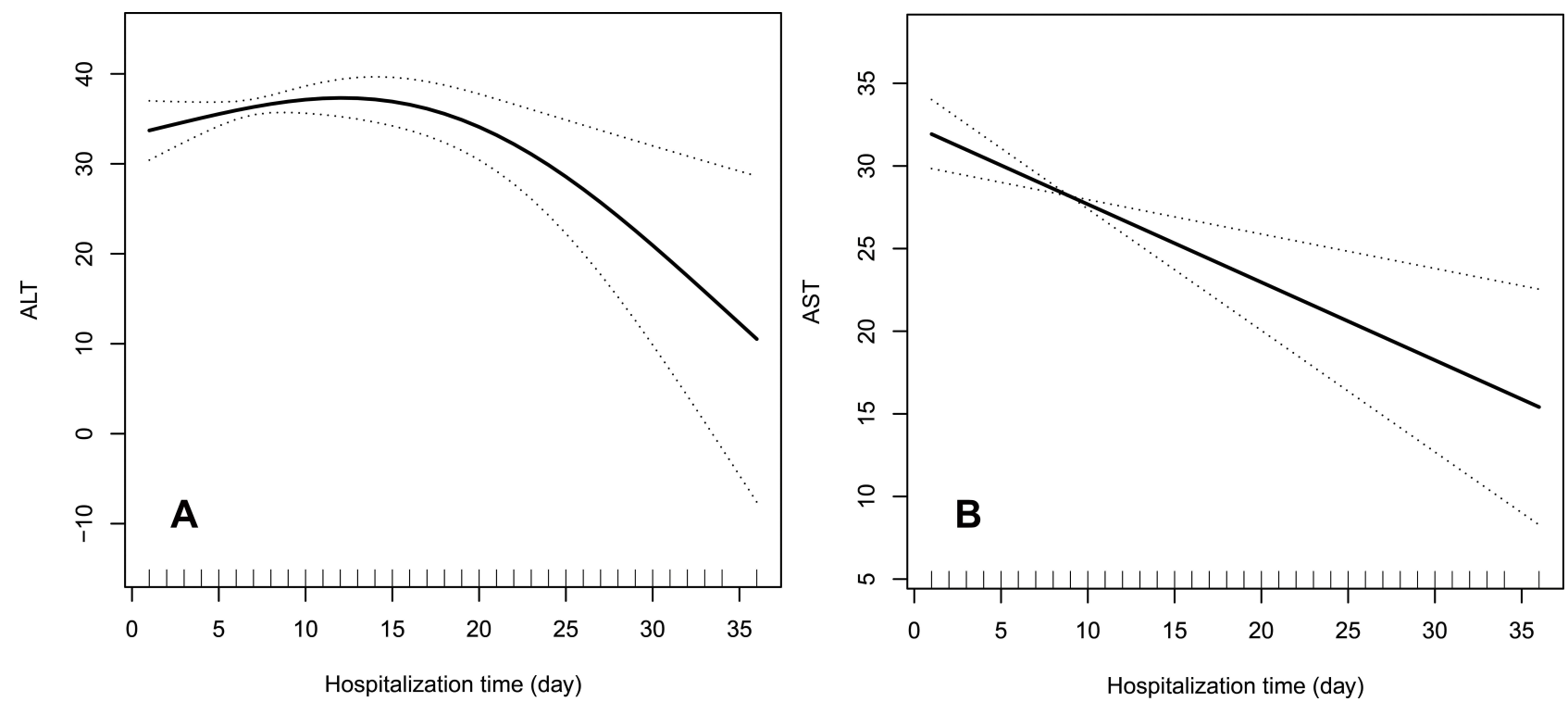

Figure I (A) Curve fitting of ALT with hospitalization time in all patients. (B) Curve fitting of AST with hospitalization time in all patients. Solid line indicates the fitted line of ALT/AST with hospitalization time; dotted line is the $95 \%$ confidence interval. Adjusted for: clinical classification, sex, age, hypertension, diabetes.

Abbreviations: ALT, alanine aminotransferase; AST, aspartate aminotransferase.
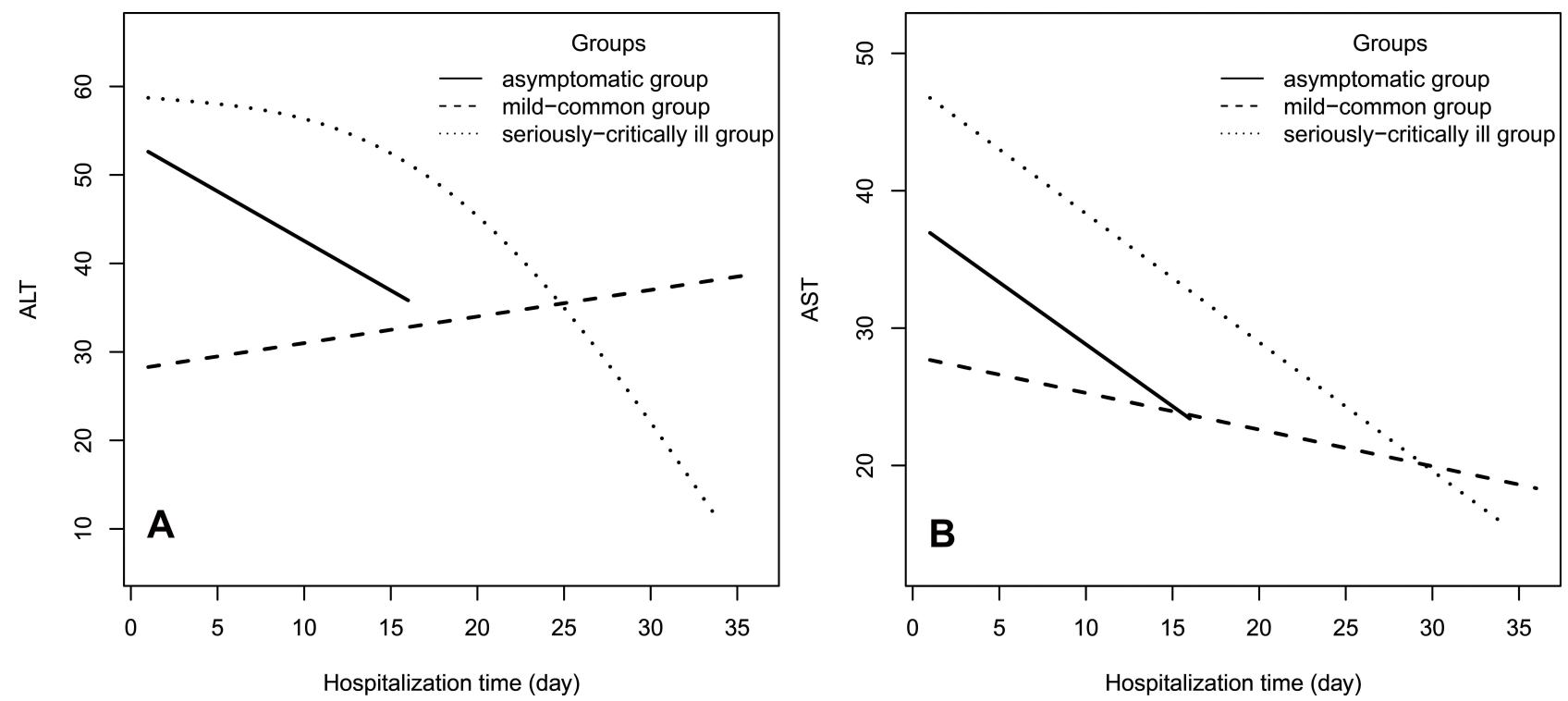

Figure 2 (A) Curve fitting of ALT with hospitalization time among asymptomatic group, mild-common group and seriously-critically ill group. (B) Curve fitting of AST with hospitalization time among asymptomatic group, mild-common group and seriously-critically ill group. Adjusted for: sex, age, hypertension, diabetes.

Abbreviations: ALT, alanine aminotransferase; AST, aspartate aminotransferase.

infection and had more severe disease. This study found that critically ill patients were older, had a higher proportion of diabetes, and had lower albumin, serum sodium, and chloride levels. $\mathrm{Xu}$ et al ${ }^{21}$ pointed out that severe COVID-19 patients exhibited a significantly higher chance of liver injury than mild patients. In the death cases of COVID-19, the incidence of liver injury can be as high as $58 \%$ or $78 \% .{ }^{22}$ In this study, although the percentage of liver injury in severe patients was significantly higher than mild patients, the relationship between liver injury and mortality could not be assessed since there was no death case.

Lei et $\mathrm{al}^{26}$ pointed out that, compared with ALT, the AST level was more frequently increased in severe patients at admission. Our study also found that the difference in AST levels at admission between different clinical types was more 
significant than ALT. In consideration of the advantages of AST, mitochondrial injury may be a potential mechanism of liver injury in severe patients. The recently published data indicated that ribosomal proteins were important hostdependent factors of SARS-CoV-2, so the virus may directly cause mitochondrial injury and increase AST levels. ${ }^{27,28}$ Several studies ${ }^{29-31}$ have shown that the angiotensin-converting enzyme 2 (ACE2) receptor mainly exists in bile duct epithelial cells, and SARS-CoV-2 can cause liver injury by binding to the ACE2 receptor; moreover, the observed liver injury is mainly from hepatocytes, and the increase in bilirubin is rarely seen. Our study also showed that even severe patients did not have significantly increased bilirubin levels, and they showed lower albumin levels. Sivandzadeh et al ${ }^{32}$ proposed that decreased serum albumin synthesis, increased catabolism, and nutritional problems are the possible causes of hypoalbuminemia in patients with severe COVID-19 and liver injury, which has been proved to be a predictor of mortality in COVID-19 patients with liver injury. ${ }^{33}$

SARS-CoV-2 infection is often associated with different degrees of abnormal liver function indexes. Current studies have shown that most COVID-19 patients have mild-to-moderate elevations in ALT/AST or both, ${ }^{20,28,34}$ especially the correlation between AST level and mortality is higher. ${ }^{26}$ The clinical evidence also shows that liver injury is common even in non-severe COVID-19 patients, ${ }^{35}$ which can be considered as a non-specific response to the general inflammation of SARS-Cov-2 infection, ${ }^{13}$ and these patients usually had a good prognosis. ${ }^{31}$ In this study, after correcting for confounding factors, we found that, as the hospitalization time prolonged, the ALT level of all patients flattened first and then gradually decreased, while the AST level decreased linearly. This observation suggested the possibility of extrahepatic AST sources. Since AST is also produced in muscles, myositis, or heart injury, likely leads to an increase in $\mathrm{AST}^{29,36}$ which reduces its liver specificity. Therefore, in the analysis of abnormal liver functions in most COVID-19 patients, ALT levels should be focused more, which is different from the view of Lei et al. ${ }^{26}$

Severe COVID-19 patients are more likely to have liver dysfunction, and the mechanism is still unclear. It is difficult to separate the independent effects of viral infection from various treatment methods, including antibiotics and the experimental antiviral drugs. ${ }^{17,30,37,38}$ Besides, these changes can be non-specific damages related to impaired immune function, infection, or hypoxia. SARS-CoV-2 infection activates the immune system, leading to reduced CD4+ and CD8+ cells $^{28,39}$ and increased secretion of proinflammatory cytokines, which then cause systemic ischemia and hypoxia. ${ }^{36}$ Hypoxia can cause liver cell death and inflammatory cell infiltration, resulting in liver injury. ${ }^{15,40-42}$ When severe liver injury occurs, we will give patients liver protection drugs. ${ }^{43,44}$ Therefore, it is necessary to monitor the levels of liver injury markers more deeply during hospitalization to develop personalized treatment plans for different patients. Since liver function indexes are not tested every day, it is particularly important to examine the changes of ALT and AST in COVID-19 patients during hospitalization. Many studies only discussed the liver enzyme indexes at admission, but our study analyzed the dynamic changes of liver function indexes in patients with different clinical types of COVID-19. As the hospitalization time increased, the changes of ALT and AST in asymptomatic patients were not significant; the ALT levels of mild-common patients did not change significantly, but AST levels significantly decreased; for seriouslycritically ill patients, the ALT levels show a significant change that first flattened and then decreased, and the daily decrease was greater than that of mild-common patients, while the change in AST was not significant. These results suggest that ALT levels can be an effective indicator to evaluate liver injury in patients with severe COVID- 19 . Therefore, we should dynamically monitor the liver function indicators of COVID-19 patients instead of focusing only on the baseline values at admission, particularly for critically ill patients. This will help with the symptomatic and supportive treatment and timely assessment of the patient condition and treatment efficacy.

This study also has some limitations. First of all, this is a retrospective, small sample size, single-center study, and it mainly focuses on mild and common patients, which could cause deviations in conclusion. Second, serum gammaglutamyl transferase (GGT) is also one of the potential markers of liver injury, which was not detected in our patients, so it was not evaluated. Third, although our results have corrected for viral hepatitis, alcoholic hepatitis, and cirrhosis to eliminate the effects of chronic liver disease, the impact of fatty liver cannot be completely ruled out; finally, because there were no deaths, the potential effect of liver function indexes on mortality cannot be assessed.

In conclusion, liver injury in COVID-19 patients is very common, especially for severe patients, and the dynamic changes of liver function indexes can provide important information for judging liver injury and evaluating the treatment 
efficacy of COVID-19 patients with different clinical types. Thus, these parameters need to be monitored during hospitalization.

\section{Data Sharing Statement}

All data generated or analyzed during this study are available from the corresponding author upon reasonable request.

\section{Ethics Approval and Informed Consent}

This study was approved by the institutional ethics committee for retrospective analysis (No. 2020-015-1), each patient signed the written informed consent, and the patients under the age of 18 signed informed consent by their legal guardian.

\section{Acknowledgments}

This study was funded by Youth Foundation Program of Nanjing Medical University (No. 2016NJMU170). The funders had no role in study design, data collection, and analysis, decision to publish, or manuscript preparation. Wei Zhao and Xiaoqing Zhang are co-first authors for this study. Feng Zhu and Xiufeng Jiang are co-corresponding authors for this study.

\section{Author Contributions}

All authors made a significant contribution to the work reported, whether that is in the conception, study design, execution, acquisition of data, analysis, and interpretation, or in all these areas; took part in drafting, revising, or critically reviewing the article; gave final approval of the version to be published; have agreed on the journal to which the article has been submitted; and agree to be accountable for all aspects of the work.

\section{Disclosure}

The authors declare that they have no conflicts of interest.

\section{References}

1. Fan Z, Chen L, Li J, et al. Clinical features of COVID-19-related liver functional abnormality. Clin Gastroenterol Hepatol. 2020;18(7):1561-1566. doi:10.1016/j.cgh.2020.04.002

2. Su TH, Kao JH. The clinical manifestations and management of COVID-19-related liver injury. J Formos Med Assoc. 2020;119(6):1016-1018. doi:10.1016/j.jfma.2020.04.020

3. Parohan M, Yaghoubi S, Seraj A. Liver injury is associated with severe Coronavirus disease 2019 (COVID-19) infection: a systematic review and meta-analysis of retrospective studies. Hepatol Res. 2020. doi:10.1111/hepr.13510

4. Cai Q, Huang D, Yu H, et al. COVID-19: abnormal liver function tests. J Hepatol. 2020;73(3):566.

5. Chen P, Zhou B. Clinical characteristics of COVID-19 in patients with liver injury. Clin Gastroenterol Hepatol. 2020;18(12):2846-2847. doi:10.1016/j.cgh.2020.04.043

6. Ye Z, Song B. Liver injury in COVID-19: diagnosis and associated factors. Liver Int. 2020;40(8):2040-2041. doi:10.1111/liv.14501

7. Cui Y, Tian M, Huang D, et al. A 55-day-old female infant infected with 2019 novel coronavirus disease: presenting with pneumonia, liver injury, and heart damage. $J$ Infect Dis. 2020;221(11):1775-1781. doi:10.1093/infdis/jiaa113

8. Hamming I, Timens W, Bulthuis ML, Lely AT, Navis G, van Goor H. Tissue distribution of ACE2 protein, the functional receptor for SARS coronavirus. A first step in understanding SARS pathogenesis. J Pathol. 2004;203(2):631-637. doi:10.1002/path.1570

9. Gu J, Han B, Wang J. COVID-19: gastrointestinal manifestations and potential fecal-oral transmission. Gastroenterology. 2020;158(6):1518-1519. doi:10.1053/j.gastro.2020.02.054

10. Huang C, Wang Y, Li X, et al. Clinical features of patients infected with 2019 novel coronavirus in Wuhan, China. Lancet. 2020;395 (10223):497-506. doi:10.1016/S0140-6736(20)30183-5

11. Cai Q, Huang D, Ou P, et al. COVID-19 in a designated infectious diseases hospital outside Hubei Province, China. Allergy. 2020;75(7):1742-1752. doi:10.1111/all.14309

12. Diao B, Wang C, Tan Y, et al. Reduction and functional exhaustion of T cells in patients with coronavirus disease 2019 (COVID-19). Front Immunol. 2020;11:827. doi:10.3389/fimmu.2020.00827

13. Li Y, Xiao SY. Hepatic involvement in COVID-19 patients: pathology, pathogenesis, and clinical implications. J Med Virol. 2020 ;92(9):1491.

14. Tian D, Ye Q. Hepatic complications of COVID-19 and its treatment. J Med Virol. 2020;92(10):1818-1824. doi:10.1002/jmv.26036

15. Portincasa P, Krawczyk M, Machill A, Lammert F, Di Ciaula A. Hepatic consequences of COVID-19 infection. Lapping or biting? Eur J Intern Med. 2020;18-24. doi:10.1016/j.ejim.2020.05.035

16. Qi X, Liu C, Jiang Z, et al. Multicenter analysis of clinical characteristics and outcomes in patients with COVID-19 who develop liver injury. $J$ Hepatol. 2020;73(2):455-458. doi:10.1016/j.jhep.2020.04.010

17. Agarwal A, Chen A, Ravindran N, To C, Thuluvath PJ. Gastrointestinal and liver manifestations of COVID-19. J Clin Exp Hepatol. 2020;10 (3):263-265. doi:10.1016/j.jceh.2020.03.001 
18. Ali N, Hossain K. Liver injury in severe COVID-19 infection: current insights and challenges. Expert Rev Gastroenterol Hepatol. 2020;14 (10):879-884. doi:10.1080/17474124.2020.1794812

19. Ali N. Relationship between COVID-19 infection and liver injury: a review of recent data. Front Med (Lausanne). 2020;7:458. doi:10.3389/ fmed.2020.00458

20. Wang Q, Zhao H, Liu LG, et al. Pattern of liver injury in adult patients with COVID-19: a retrospective analysis of 105 patients. Mil Med Res. 2020;7(1):28. doi:10.1186/s40779-020-00256-6

21. Xu L, Liu J, Lu M, Yang D, Zheng X. Liver injury during highly pathogenic human coronavirus infections. Liver Int. 2020;40(5):998-1004. doi:10.1111/liv.14435

22. Mao R, Qiu Y, He JS, et al. Manifestations and prognosis of gastrointestinal and liver involvement in patients with COVID-19: a systematic review and meta-analysis. Lancet Gastroenterol Hepatol. 2020;5(7):667-678. doi:10.1016/S2468-1253(20)30126-6

23. Diagnosis and Treatment Protocol for Novel Coronavirus Pneumonia (Trial Version. 7). Chin Med J (Engl). 2020;133(9):1087-1095. doi:10.1097/ CM9.0000000000000819

24. Wood SN. Low-rank scale-invariant tensor product smooths for generalized additive mixed models. Biometrics. 2006;62(4):1025-1036. doi: $10.1111 /$ j.1541-0420.2006.00574.x

25. Kukla M, Skonieczna-Zydecka K, Kotfis K, et al. COVID-19, MERS and SARS with Concomitant Liver Injury-Systematic Review of the Existing Literature. J Clin Med. 2020;9:5. doi:10.3390/jcm9051420

26. Lei F, Liu YM, Zhou F, et al. Longitudinal association between markers of liver injury and mortality in COVID-19 in China. Hepatology. 2020;72 (2):389.

27. Bloom PP, Meyerowitz EA, Reinus Z, et al. Liver biochemistries in hospitalized patients with COVID-19. Hepatology. 2020;73(3):890-900.

28. Wang Y, Liu S, Liu H, et al. SARS-CoV-2 infection of the liver directly contributes to hepatic impairment in patients with COVID-19. J Hepatol. 2020;73(4):80.

29. Phipps MM, Barraza LH, LaSota ED, et al. Acute liver injury in COVID-19: prevalence and association with clinical outcomes in a large US Cohort. Hepatology. 2020;72(3):807-817. doi:10.1002/hep.31404

30. Boeckmans J, Rodrigues RM, Demuyser T, Pierard D, Vanhaecke T, Rogiers V. COVID-19 and drug-induced liver injury: a problem of plenty or a petty point? Arch Toxicol. 2020;94(4):1367-1369. doi:10.1007/s00204-020-02734-1

31. Xie H, Zhao J, Lian N, Lin S, Xie Q, Zhuo H. Clinical characteristics of non-ICU hospitalized patients with coronavirus disease 2019 and liver injury: a retrospective study. Liver Int. 2020;40(6):1321-1326. doi:10.1111/liv.14449

32. Sivandzadeh GR, Askari H, Safarpour AR, et al. COVID-19 infection and liver injury: clinical features, biomarkers, potential mechanisms, treatment, and management challenges. World J Clin Cases. 2021;9(22):6178-6200. doi:10.12998/wjcc.v9.i22.6178

33. Zhang Y, Zheng L, Liu L, Zhao M, Xiao J, Zhao Q. Liver impairment in COVID-19 patients: a retrospective analysis of 115 cases from a single centre in Wuhan city, China. Liver Int. 2020;40(9):2095-2103. doi:10.1111/liv.14455

34. Debes JD, Anugwom CM, Aby ES. Systematic analysis of acute liver injury during SARS-CoV-2 infection. Dig Liver Dis. 2020;52(9):953-955. doi:10.1016/j.dld.2020.05.006

35. Garrido I, Liberal R, Macedo G. Review article: COVID-19 and liver disease-what we know on 1st May 2020. Aliment Pharmacol Ther. 2020;52 (2):267-275. doi:10.1111/apt.15813

36. Alqahtani SA, Schattenberg JM. Liver injury in COVID-19: the current evidence. United European Gastroenterol J. 2020;8(5):509-519. doi: $10.1177 / 2050640620924157$

37. Lee IC, Huo TI, Huang YH. Gastrointestinal and liver manifestations in patients with COVID-19. J Chin Med Assoc. 2020;83(6):521-523. doi:10.1097/JCMA.0000000000000319

38. Muhovic D, Bojovic J, Bulatovic A, et al. First case of drug-induced liver injury associated with the use of tocilizumab in a patient with COVID-19. Liver Int. 2020;40(8):1901-1905. doi:10.1111/liv.14516

39. Wang H, Qiu P, Liu J, Wang F, Zhao Q. The liver injury and gastrointestinal symptoms in patients with Coronavirus Disease 19: a systematic review and meta-analysis. Clin Res Hepatol Gastroenterol. 2020;44(5):653-661. doi:10.1016/j.clinre.2020.04.012

40. Feng G, Zheng KI, Yan QQ, et al. COVID-19 and liver dysfunction: current insights and emergent therapeutic strategies. J Clin Transl Hepatol. 2020;8(1):18-24. doi:10.14218/JCTH.2020.00018

41. Li J, Fan JG. Characteristics and mechanism of liver injury in 2019 Coronavirus disease. J Clin Transl Hepatol. 2020;8(1):13-17. doi:10.14218/ JCTH.2020.00019

42. Kunutsor SK, Laukkanen JA. Markers of liver injury and clinical outcomes in COVID-19 patients: a systematic review and meta-analysis. $J$ Infect. 2020;82(1):159

43. Zhang C, Shi L, Wang FS. Liver injury in COVID-19: management and challenges. Lancet Gastroenterol Hepatol. 2020;5(5):428-430. doi:10.1016/S2468-1253(20)30057-1

44. Wu J, Song S, Cao HC, Li LJ. Liver diseases in COVID-19: etiology, treatment and prognosis. World J Gastroenterol. 2020;26(19):2286-2293. doi:10.3748/wjg.v26.i19.2286

International Journal of General Medicine

Dovepress

\section{Publish your work in this journal}

The International Journal of General Medicine is an international, peer-reviewed open-access journal that focuses on general and internal medicine, pathogenesis, epidemiology, diagnosis, monitoring and treatment protocols. The journal is characterized by the rapid reporting of reviews, original research and clinical studies across all disease areas. The manuscript management system is completely online and includes a very quick and fair peer-review system, which is all easy to use. Visit http://www.dovepress.com/testimonials.php to read real quotes from published authors.

Submit your manuscript here: https://www.dovepress.com/international-journal-of-general-medicine-journal 